\title{
Bulk Growth, Structural and Optical Properties of Pure and Doped 1, 3, 5-Triphenylbenzene Crystal by Sankaranarayanan-Ramasamy method for Scintillator Applications
}

\author{
S. KALAINATHAN* ${ }^{*}$ N. DURAIRAJ* and R. KUMAR* \\ ${ }^{*}$ Centre for Crystal Growth, VIT University, Vellore, Tamilnadu-632014, India \\ "Nuclear and Radio-analytical Chemistry Section, FChD, MC \& MFCG, IGCAR, Kalpakkam-603102, India \\ E-mail:kalainathan@yahoo.com
}

\begin{abstract}
The present work is elaborate unidirectional bulk growth and characterization of 1,4-diphenyl-1,3butadiene (DPB) and 2,5-diphenyloxazole (PPO) doped 1,3,5-triphenylbenzene (3PB) crystal from solution by unidirectional Sankaranarayanan-Ramasamy (SR) method. The investigation of XRD, UV-Vis-NIR absorption and transmittance studies were carried out for the pure and DPB, PPO doped 3PB crystals. The lattice parameters and phase analysis of doped crystals by XRD were confirmed orthorhombic crystal structure.The distortion in XRD and UV-Vis-NIR transmission spectra reveals that the incorporation of dopant molecules in $3 \mathrm{~PB}$.
\end{abstract}

Keywords : Organic Scintillator crystals, SR growth technique, Doped Organic Scintillator

\section{INTRODUCTION}

Scintillation counting is a highly sensitive technique to detect the invisible radiations. In $21^{\text {st }}$ century number of new organic scintillator crystal was grown and carried out the radiation detection analysis [1]. The particle discrimination like alpha, beta, gamma and neutron separation in complicated radioactivity field is possible by two decay (prompt and delayed) fluorescence of organic molecules. Some of the organic molecules were existing two decay fluorescence, which was also grown by a single crystal and demonstrated for the neutron-gamma discrimination in high energy radiation field [2, 3]. Nowadays it is considered to industrialize in several applications such as border security and environmental control systems, as well as in radiation medicines $[4,5]$. However, the conventional crystal growth system has a number of unavoidable drawbacks, for example, high production cost, specialized equipment required for its production as well as difficulties in obtaining a large size of scintillators. Hence overcome this issues many researchers have found new organic scintillators and grown the larger single crystals with low-cost techniques [6-8]. $3 \mathrm{~PB}$ is one of the promising organic scintillator crystals which give the better pulse shape for neutron and gamma discrimination [8]. The present authors also have grown the bulk size unidirectional 3PB single crystal by SankaranarayananRamasamy (SR) technique reported decay time and other preliminary studies for device fabrication [9].

For the scintillator application, larger light output and shorter decay time would be most expected, since it covers the large events of radiation detection [10]. But 3PB has lower light output value and longer decay time compares to existing material $[8,9]$. Hence, Zaitseva et al. reported the small addition PPO molecule in 3PB enhanced the luminescence intensity [8]. Adding dopant in a core compound will increase the density of triplet energy level which improves delayed fluorescence and decreasing the decay time. Since triplets are known to be mobile in organic compounds, the energy migrates until two triplets collide and annihilate to form one singlet excited state. The lifetime of the delayed emission is determined by the lifetime of triplet energy level and the rate of collisions among them. In two decay emission, prompt fluorescence corresponds to direct de-excitation of singlet energy level transition and delayed emission is due to collisional interaction pairs of lowest excited triplet state of the molecules. The important of the collisional interaction of triplet energy level is the identification of neutron detection in an organic scintillator [11]. The quantity of dopants in a core molecule is necessary because there may be chances to suppress the luminescence intensity. Triplet energy level will be enhanced in a core molecule during the addition of a defined quantity of dopants. The mobility of triplet states collide and annihilate to form the singlet states will enhance the luminescence intensity and decreases the decay time. However, Zaitseva et al. not quantified the addition of PPO dopant in 3PB. An attempt was made by Balamurugan et al., to enhance fluorescence intensity of naphthalene crystal by doping the 2,5-diphenyloxazole (PPO) fluorophore. Also, they have demonstrated the limitation of dopant in host molecule. Four different concentration 0.01, 0.03, 0.05 , and $0.07 \mathrm{~mol} \%$ of PPO doped in naphthalene and analyzed the fluorescence emission intensity and decay time. Among the four concentrations, the emission intensity was increased at 0.01 and $0.03 \mathrm{~mol} \%$. But the maximum emission intensity was observed at $0.03 \mathrm{~mol} \%$. Further increasing the concentration of dopant $0.03 \mathrm{~mol} \%$, the emission intensity was decreased due to concentration quenching effect [12]. N.Z.Gulunov et al., described 
the dopant 1,4-diphenyl-1,3-butadiene (DPB) distribution in p-terphenyl. They have analyzed the DPB distribution from 0.1 to 0.7 mass $\%$ in p-terphenyl. The maximum light output values were observed in the range of $0.1-0.3 \%$ above this concentration light output decreased due to concentration quenching effect [13]. Recently Qing Ai et al., reported the pentacene doped p-terphenyl crystal by vertical Bridgman technique using double walled ampoule [14]. Based on the above literature, the present authors were doped the DPB and PPO in $3 \mathrm{~PB}$. The different concentrations $0.01,0.03,0.05$ and $0.07 \mathrm{~mol} \%$ of dopants were analyzed to enhanced fluorescence emission and decay time was reported [15]. Due to the low level impurities, the final amount of concentration was unable to measure in doped crystals it may be same or less than the initial concentration of dopants. The accommodation of dopant is also not clear at this stage. The guest molecules maybe randomly filled in the vacancy or defects in the host crystal lattices $[12-14,16]$.The present work is to elaborate unidirectional bulk growth and the DPB and PPO dopants incorporation in $3 \mathrm{~PB}$.

\section{EXPERIMENTAL TECHNIQUE}

\subsection{Crystal Growth}

The authors were reported the solubility and bulk growth of pure 3PB single crystal by conventional solution growth and SR technique [9]. The optimized concentration ratio of $0.01 \mathrm{~mol} \%$ $\mathrm{DPB}$ and $0.05 \mathrm{~mol} \% \mathrm{PPO}$ added in $3 \mathrm{~PB}$ [15]. At $35^{\circ} \mathrm{C}, 300 \mathrm{ml}$ bulk saturated solution was prepared in tetrahydrofuran solvent. A suitable seed crystal ( $9 \mathrm{~mm}$ diameter) cut along (100) direction was prepared. The prepared seed crystal was mounted in a $300 \mathrm{~mm}$ length $25 \mathrm{~mm}$ diameter borosilicate glass ampoule. The prepared saturated solution was filled in a glass ampoule and tightly covered with aluminum foil to avoid the uncontrollable solvent evaporation. The saturated solution filled in a glass ampoule was

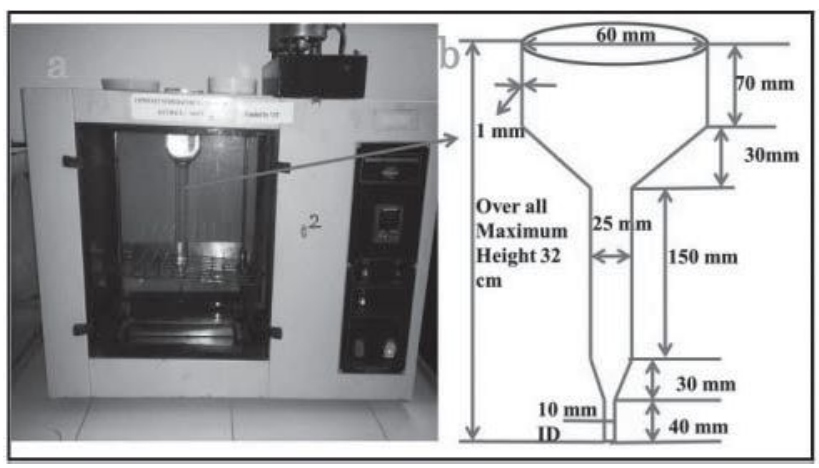

Figure 1 (a) Constant temperature water bath (b) glass ampoule

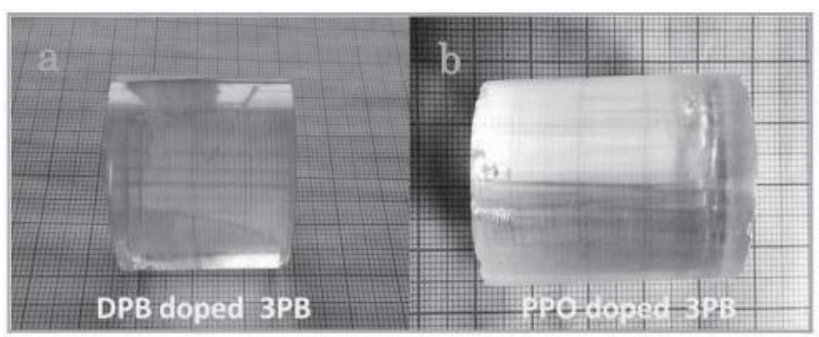

Figure 2 Cut and polished (a) DPB doped 3PB (b) PPO doped 3PB crystals kept in a constant temperature water bath (accuracy \pm 0.01 ) and the temperaturewas fixed at $35^{\circ} \mathrm{C}$. To prevent seed dissolving in saturated solution the bath temperature was reduced to $34^{\circ} \mathrm{C}$ at the rate of $0.25^{\circ} \mathrm{C}$ every $2 \mathrm{hrs}$. Constant temperature water bath and glass ampoule were shown Figure 1b. A small pinhole was made of the aluminum foil to evaporate the solvent. After three days, due to the continuous evaporation of the solvent, the solution has attained the supersaturation then the seed crystal was started to grow. After 47 days the, bulk size ( $25 \mathrm{~mm}$ diameter) DPB and PPO doped $3 \mathrm{~PB}$ crystals were harvested. The cut and polished crystals were shown in Figure 2.

\subsection{Instruments for characterization}

The cell parameters of pure and doped 3PB crystals were calculated at room temperature using Bruker Kappa APEX-II with Mo $\mathrm{K} \alpha$ radiation $(\lambda=0.7107 \AA$ ). The fine powder of $3 \mathrm{~PB}$ crystal was used for phase analysis and crystalline nature. The powder $\mathrm{X}$-ray diffraction was carried out by Bruker X-ray diffractometer with $\mathrm{CuK}_{\alpha}$ radiation $(\lambda=1.5406 \AA)$ scanning angles ranging from 5 to $45^{\circ}$ were used with scan rate $0.02^{\circ} \mathrm{s}^{-1}$. The diffraction of different planes at the specific $2 \theta$ angles was indexed by Powder X software [17]. The optical absorption and transmittance studies were analyzed by using the ELICO double beam UV-VisNIR spectrometer (SL-218) in the region of 200-1100 nm. Wellpolished $2 \mathrm{~mm}$ thin pure and doped crystals are used for the optical absorption and transmission studies.

\section{RESULT AND DISCUSSION}

\subsection{X-Ray Diffraction Analysis}

The powder XRD patterns of DPB and PPO doped 3PB was compared with pure $3 \mathrm{~PB}$ crystal which is shown in Figure 3. There is no additional peak or phase changes were observed in the doped crystals spectrum which reveals no change in the structure of pure $3 \mathrm{~PB}$. The peak broadening can be attributed to lattice strain due to dopants. It inferred that the dopants molecules included in lattices. The differences in peak intensity can be attributed to the orientation of grains and difference in sizes. Due to the lower amount of dopants concentrations, the accommodation of dopant is not clear in doped crystals. The guest molecules are randomly filled in the

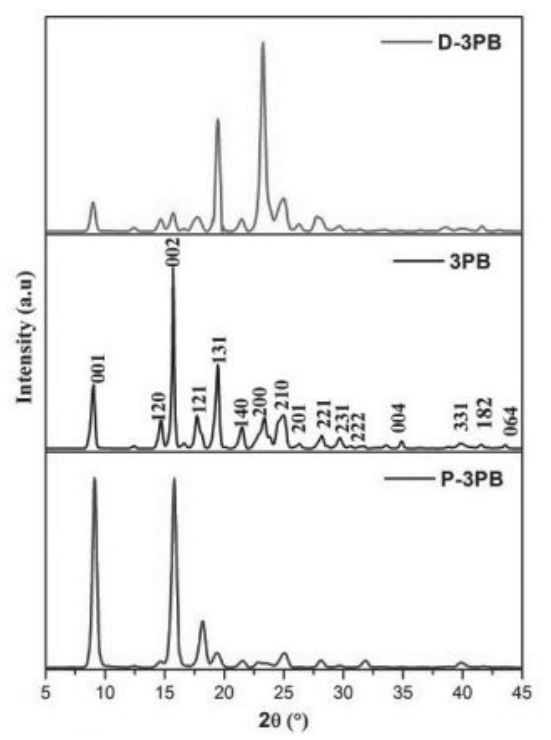

Figure 3 XRD spectrum of Pure and doped 3PB 
Table 1 comparison of cell parameters of pure and doped crystals

\begin{tabular}{cccc}
\hline $\begin{array}{c}\text { Lattice } \\
\text { parameters }\end{array}$ & $\begin{array}{c}3 \mathrm{~PB} \\
{[9]}\end{array}$ & $\begin{array}{c}\mathrm{D}-3 \mathrm{~PB} \\
\text { [present } \\
\text { work] }\end{array}$ & $\begin{array}{c}\text { P-3PB } \\
\text { [present } \\
\text { work] }\end{array}$ \\
\hline $\mathrm{a}(\AA)$ & 7.603 & 7.634 & 7.645 \\
\hline $\mathrm{b}(\AA)$ & 19.782 & 19.84 & 19.94 \\
\hline $\mathrm{C}(\AA)$ & 11.296 & 11.308 & 11.347 \\
\hline Angles & $\alpha=\beta=\gamma=90^{\circ}$ & $\alpha=\beta=\gamma=90^{\circ}$ & $\alpha=\beta=\gamma=90^{\circ}$ \\
\hline Space group & $\mathrm{Pna}_{1}$ & $\mathrm{Pna}_{1}$ & Pna $2_{1}$ \\
\hline
\end{tabular}

vacancy or defects in the host crystal lattices [14]. It may be a chance of disorder in the $3 \mathrm{~PB}$ crystal lattices. Therefore the lattice parameters of doped crystals D-3PB and P-3PB raises further increasing dopant concentrations. Samples with low content of impurity may be reaching the equilibrium, becomes more regular. The cell parameters and space group for pure and doped crystals were listed in Table 1. The observed values confirm the doped crystals also belongs to an orthorhombic structure. The calculated cell parameters and observed peak patterns are good agreement with JCPDS card no. 33-1943 [18].

\subsection{UV-Vis Analysis}

The absorption and transmission spectra of DPB and PPO doped crystals shown in Figure 4 (a\&b). The absorption edges are same in all dopants. The same behavior was observed in pure and doped crystals over the visible and NIR region. The absorptions are due to the $\pi-\pi^{*}$ transition of individual molecules. The distortion in transmission spectra (figure iii b) of doped crystals reveals the incorporation of dopants in $3 \mathrm{~PB}$.
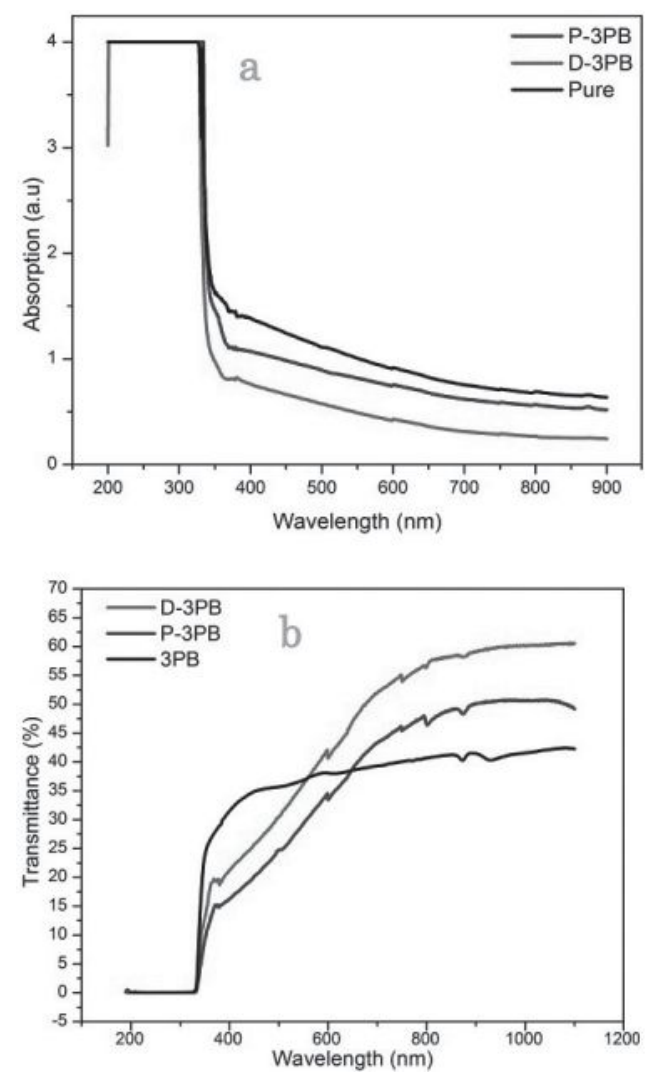

Figure 4 (a) UV-Vis absorption (b) Transmittance of Spectrum pure and doped 3PB crystal.

\subsection{FTIRSpectral Analysis}

Figure 5 reveals the infrared spectra for pure and doped 3PB. There is no peak shift and peaks observed from pure 3PB to doped crystals which explains that the functional groups are not sensitive to the low-level concentrations [19]. The FTIR wavenumbers and the vibrational assignments of pure and doped 3PB crystals were listed in Table 2.

\section{CONCLUSIONS}

The DPB, PPO doped unidirectional bulk size ( $25 \mathrm{~mm}$ diameter) $3 \mathrm{~PB}$ crystals were grown in solution by SR technique. The unit cell parameters of pure and DPB and PPO doped 3PB crystals were calculated which shows the increasing dopant ratio 0.01 and

Table 2 Functional group assignments of pure and DPB, PPO doped 3PB crystals

\begin{tabular}{|c|c|c|c|c|}
\hline \multirow[t]{6}{*}{ S.No. } & \multicolumn{3}{|c|}{ Wavenumber $\left(\mathrm{cm}^{-1}\right)$} & \multirow{6}{*}{$\begin{array}{l}\text { Vibrational } \\
\text { Assignment }\end{array}$} \\
\hline & Pure & $0.01 \mathrm{~mol} \%$ & 0.05 & \\
\hline & 3PB & DPB doped & $\mathrm{mol} \%$ & \\
\hline & & $3 \mathrm{~PB}$ & PPO & \\
\hline & & & doped & \\
\hline & & & $3 \mathrm{~PB}$ & \\
\hline \multirow[t]{2}{*}{1} & 3055.24 & 3057.17 & 3057.17 , & C-H \\
\hline & 3030.17 & 3032.10 & 3032.10 & Stretching \\
\hline \multirow[t]{2}{*}{2} & 1591.27, & 1593.20 & 1593.20 & $\mathrm{C}=\mathrm{C}$ \\
\hline & 1494.83 & 1494.83 & 1494.83 & $\begin{array}{l}\text { stretching in } \\
\text { phenyl ring }\end{array}$ \\
\hline \multirow[t]{6}{*}{3} & 1072.35 & 1074.35, & 1074.35, & Phenyl ring \\
\hline & 1028.13 & 1026.13 & 1026.13 & C-H \\
\hline & & & & stretching \\
\hline & & & & in-plane \\
\hline & & & & bending \\
\hline & & & & vibration \\
\hline \multirow[t]{4}{*}{4} & 746.46 & 746.45 & 746.45 & Phenyl ring \\
\hline & 690.52 & 688.59 & 690.52 & C-H out-plane \\
\hline & & & & bending \\
\hline & & & & vibration \\
\hline \multirow[t]{6}{*}{5} & 869.90 & 869.90 & 869.90 & 1,3-meta \\
\hline & & & & position \\
\hline & & & & substitution \\
\hline & & & & of the \\
\hline & & & & phenyl \\
\hline & & & & groups. \\
\hline
\end{tabular}

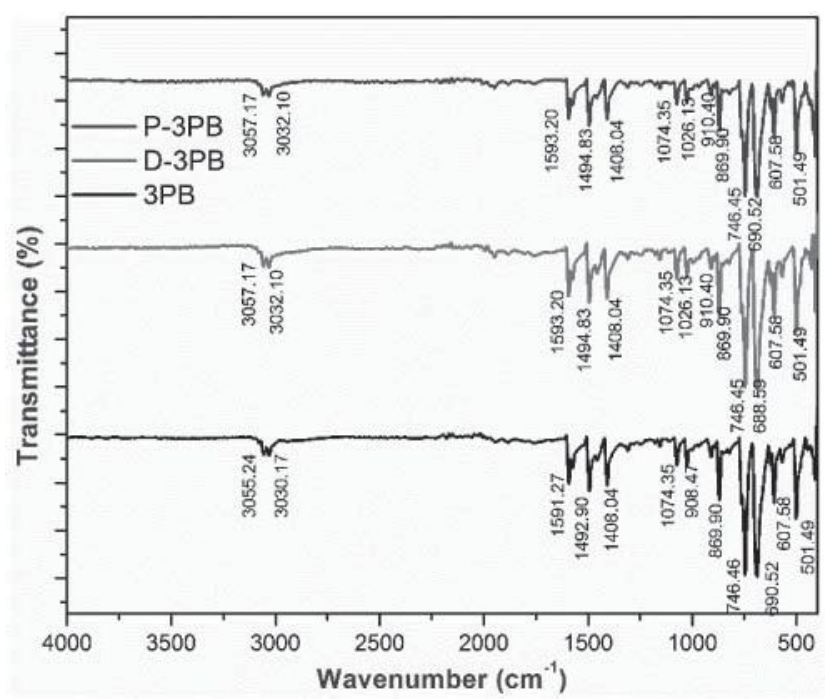

Figure 5 FTIR spectra of pure 3PB crystal and DPB and PPO crystals. 
$0.05 \mathrm{~mol} \%$ the slight changes in the lattice parameters. But there is no new phase formation or the phase changes in the XRD pattern. The Difference in peak intensity peak broadening reveals the lattice strain due to the addition of dopants. In the optical analysis, same absorption edges were observed, but distortion in transmission spectra is confirming the dopants attributed in the pure $3 \mathrm{~PB}$ crystal. The FTIR spectral features for doped crystals about the corresponding spectral feature of pure 3PB. Hence XRD and FTIR spectra show that the doping such small amount does not lead noticeable changes of intermolecular interactions.

\section{Acknowledgement}

The authors thank the DAE-BRNS for providing financial support(sanction no. 34/14/22/2014-BRNS0294 dated 16 May 2014) and VIT University management for their constant encouragement.

\section{References}

[1] C.R. Ronda, Luminescence: From Theory to Applications, Wiley-VCH, Weinheim, (2008).

[2] N. Zaitseva, J. Newby, S. Hamel, L. Carman, M. Faust, V. Lordi, N. Cherepy, W. Stoeffl, S. Payne, Neutron detection with single crystal organic scintillators, https://e-reports-ext. llnl.gov/pdf/375697.pdf

[3] S.Selvakumar, K.Sivaji, A.Arulchakkaravarthi, S.Sankar, Enhanced fluorescence and time resolved fluorescence properties of p-terphenyl crystal grown by selective selfseeded vertical Bridgman technique, Materials Letters 61, 4718-4721(2007).

[4] http://www.cryosbeta.kharkov.ua/organic.php

[5] www.inradoptics.com, White paper, Production of stilbene for fast neutron detection

[6] G.Hull, Natalia P. Zaitseva, Nerine J. Cherepy, Jason R. Newby, Wolfgang Stoeffl, and Stephen A. Payne, IEEE Transactions On Nuclear Science, 56, 899-903(2009).

[7] Edgar V. van Loef, Jarek Glodo, Urmila Shirwadkar, Natalia Zaitseva, Kanai S. Shah, Novel Organic Scintillators for Neutron Detection, IEEE, 1007-1009(2010).

[8] Natalia Zaitseva, Leslie Carman, Andrew Glenn, Jason Newby, Michelle Faust, Sebastien Hamel,NerineCherepy, Stephen Payne, Application of solution techniques for rapid growth of organic crystals, Journal of Crystal Growth 314, 163-170(2011).

[9] N.Durairaj, S.Kalainathan, M.V.Krishnaiah, Investigation on unidirectional growth of 1,3,5-Triphenylbenzene by Sankaranarayanan-Ramasamy method and its characterization of lifetime, thermal analysis, hardness and etching studies, Material Chemistry and Physics, 181, 529-537(2016).

[10] G. F. Knoll, Radiation Detection and Measurement, 3rd Ed. New York: Wiley, (2000).

[11] Natalia Zaitseva, Andrew Glenn, Leslie Carman, Robert Hatarik, Sebastien Hamel, Michelle Faust,Brandon Schabes, NerineCherepy, and Stephen Payne, Pulse Shape Discrimination in Impure and MixedSingle-Crystal Organic Scintillators, IEEE Transactions On Nuclear Science, 58 (6), 3411-3420, (2011).

[12] N.Balamurugan, A.Arulchakkaravarthi, P.Ramasamy, Growth of 2,5-diphenyloxazole-doped naphthalene crystal by Bridgman method and its fluorescence studies, Journal of Crystal Growth 310, 2115-2119 (2008).

[13] Nikolai Z. Galunov, Igor V. Lazarev, Eugenia V. Martynenko, Valery V. Vashchenko\& Elena V. Vashchenko, Distribution Coefficient of 1,4-diphenyl-1,3- butadiene in p-terphenyl Single Crystal and Its Influence on Scintillation Crystal Light Output, Molecular Crystals and Liquid Crystals, 616:1, 176186, (2015). DOI:10.1080/15421406.2014.991105.

[14] Qing Ai, Peifeng Chen, Yuxiang Feng, Yebin Xu, Growth of Pentacene-Doped $\mathrm{p}$ Terphenyl Crystals by Vertical Bridgman Technique and Doping Effect on Their Characterization, Cryst. Growth Des. 17, 2473-2477 (2017).

[15] [15] N. Durairaj, S. Kalainathan, R. Kumar, Fluorescence Emission and Decay Time Studies on Doped 1, 3, 5-Triphenylbenzene Scintillator Crystal Grown by Solution Growth Technique. Mechanics, Materials Science \& Engineering, 9 (2), 220-221 (2017).

[16] Mullin, J. W. Crystallization, Butterworth-Heinemann:Oxford, U.K., 4th ed., 205-284, 2001;

[17] H. Milledge, et al., University College London, England, ICDD, Grant in eAid, (1982).

[18] W. Kaminsky, J. Appl. Cryst. 40, 382(2007).

[19] Amorim da Costa, A. M.; Amado, A. M. Solid State Ionics, 125, 263-269, (1999). 